\title{
Matratzenüberzüge für Hausstauballergiker geprüft
}

\begin{abstract}
Zu den präventiven Strategien bei Patienten mit Hausstaubmilbenallergie zählt als wesentlicher Bestandteil die Verwendung allergenundurchlässiger Matratzenummantelungen - sog. Encasings. Diese sollen verhindern, daß aus dem Inneren oder von der Oberfläche der Matratze allergenhaltige Partikel freigesetzt werden und den Patienten während der Bettruhe belasten.
\end{abstract}

D er Grad, zu dem die verwendeten Encasing-Materialien Allergenpartikel passieren lassen, ist somit entscheidend für deren Qualität. Zugleich müssen die Bezüge aber ausreichend durchlässig für Wasserdampf sein. Andernfalls sammelt sich zu viel Flüssigkeit im Bett an, was sich äußerst ungünstig auf

Tab. 1: Verschlüsselung der Produkte.

Proben-Nummer I:

Proben-Nummer II:

Proben-Nummer III:

Proben-Nummer IV:

Proben-Nummer V:

Proben-Nummer VI:

Proben-Nummer VII:

Proben-Nummer VIII:

Proben-Nummer IX:

Proben-Nummer $\mathrm{X}$ : den Schlafcomfort und damit auf die Compliance der Patienten auswirkt.

Inwieweit die zahlreichen angebotenen Encasings in Deutschland diesen beiden Anforderungen gerecht werden, ist jetzt erstmals in einer Studie untersucht worden.

Neun Encasing-Materialien verschiedener Hersteller sowie eine zusätzliche Materialprobe wurden von zwei unabhängigen Instituten auf ihre Partikel- und Wasserdampfdurchlässigkeit analysiert. Den Untersuchern war dabei weder das $\mathrm{Ma}$ terial noch der Hersteller bekannt. Die Produkte waren wie in Tabelle 1 angegeben verschlüsselt.

Um die Partikeldurchlässigkeit festzustellen, wurden zwei verschie- dene Prüfluftgemische (Außenluft und Kohle-Feinstaub) mit einer eigens für diese Studie entwickelten Apparatur an die Probenmaterialien angesogen und deren Partikeldurchgang durch einen hinter dem Encasing-Material angebrachten Filter gemessen. Die Bewertung beruhte zum einen auf der maximalen durchgelas-
Allergy Control „Perfect“ (Dr. Beckmann)

Allergy Control „Original“ (Dr. Beckmann)

Alprotec (HAL-Allergie)

Intervent (Gore; seit 1.10.96 nicht mehr auf dem Markt)

Bencase (früher Bencard, jetzt PSP)

Allergocover (Allergopharma)

Med-tex (INNOVALL)

Muster eines japanischen Herstellers

Med-tex Super (INNOVALL)

Cotton Guard (NAS/USA) senen Partikelgröße, zum anderen auf der Zahl der passierten Partikel in den beiden Versuchsserien.

Die Wasserdampfdurchlässigkeit wurde mit dem sog. Thermoregulationsmodell ermittelt. Bei diesem
Normverfahren handelt es sich um eine Art Klimaschrank, der die Feuchtigkeitsabgabe der Haut bei bestimmten Temperaturen simuliert. Für die Untersuchung wurde das Modell mit den jeweiligen Materialien bedeckt und der sog. Wasserdampfdurchgangswiderstand gemessen.

Eine zusammenfassende Bewertung der Partikel- und der Wasserdampfdurchlässigkeit ist der Tabelle 2 zu entnehmen.

Die Gegenüberstellung zeigt, daß die Partikeldurchlässigkeit nicht mit der Feuchtigkeitsdurchlässigkeit korreliert. So erreichte keine der Proben das Prädikat ,sehr gut" in beiden Kategorien. Bei der Verordnung von Encasings muß also abgewogen werden zwischen 1. der aus allergologischer Sicht entscheidenden Partikeldurchlässigkeit und 2. der für den Schlafkomfort wichtigen Wasserdampfdurchlässigkeit.

Die vorliegende Untersuchung soll als Anhaltspunkt zur Beratung des Patienten dienen und zugleich zu einer wissenschaftlich fundierten Diskussion beitragen. Sie ist kein Ersatz für eine klinische Untersuchung. Weitere wichtige Parameter wie physikalische Merkmale oder Haltbarkeit der Encasings konnten in der Studie nicht berücksichtigt werden, müßten aber in eine umfassende Bewertung einfließen.

Die zum Teil sehr unterschiedlichen Preise der verschiedenen Produkte sind nach Ansicht der Autoren auf jeden Fall kein ausreichendes Kriterium, die Qualität des Bezuges zu beurteilen.

$(z o)$

Quelle: Kainka E, Umbach K-H, Müsken H. Encasing-Bezüge: Untersuchungen zum Staubrückhaltevermögen und zur Wasserdampfdurchlässigkeit. Pneumologie 1997; 51: 2-9.

Tab. 2: Bewertung der Produkte nach Partikel- und Wasserdampfdurchlässigkeit.

\begin{tabular}{|cll|}
\hline Proben-Nr. & Staubrückhaltung & Wasserdampfdurchlässigkeit \\
\hline I & sehr gut & gut \\
II & gut & gut \\
III & unbefriedigend & sehr gut \\
IV & gut & sehr gut \\
V & gut & unbefriedigend \\
VI & unbefriedigend & sehr gut \\
VII & sehr gut & unbefriedigend \\
VIII & unbefriedigend & sehr gut \\
IX & sehr gut & gut \\
X & gut & unbefriedigend \\
\hline
\end{tabular}

